\title{
Good Research Practice: Lessons from Animal Care and Use
}

\author{
Javier Guillén and Thomas Steckler
}

\section{Contents}

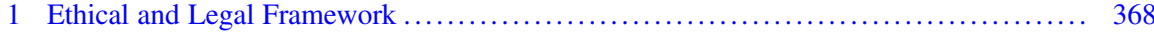

1.1 Recommendations for the Care and Use of Laboratory Animals .............. 369

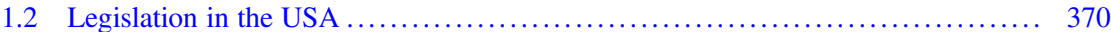

1.3 Legislation in the European Union ................................... 370

1.4 Legislation in Other Countries ........................................ 371

2 Implications for Preclinical Data Quality ..................................... 372

2.1 Oversight Bodies Impact on Preclinical Data Quality ......................... 372

2.2 Animal Care and Use Programs Affect Preclinical Data Quality ............... 372

2.3 Health Status Influencing Preclinical Data ............................. 373

2.4 The Impact of Housing and Husbandry . . . . . . . . . . . . . . . . . . . . . . . . 374

3 Assessment of Animal Care and Use Programs ............................ 375

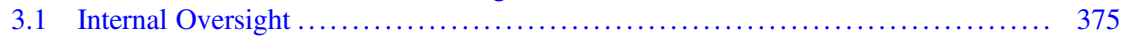

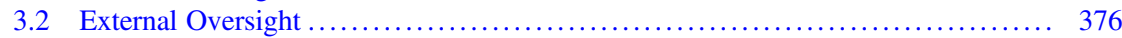

3.3 The AAALAC International Accreditation Process ....................... 376

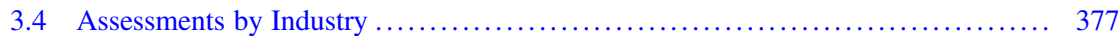

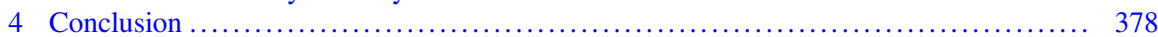

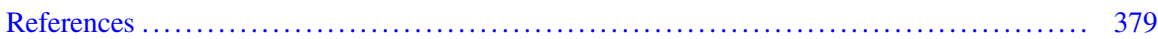

\begin{abstract}
Animal care and use play a pivotal role in the research process. Ethical concerns on the use of animals in research have promoted the creation of a legal framework in many geographical areas that researchers must comply with, and professional organizations continuously develop recommendations on specific areas of laboratory animal science. Scientific evidence demonstrates that many aspects of animal care and use which are beyond the legal requirements have direct impact
\end{abstract}

\section{J. Guillén}

AAALAC International, Pamplona, Spain

T. Steckler $(\bowtie)$

Janssen Pharmaceutica NV, Beerse, Belgium

e-mail: TSTECKLE@its.jnj.com 
on research results. Therefore, the review and oversight of animal care and use programs are essential to identify, define, control, and improve all of these aspects to promote the reproducibility, validity, and translatability of animal-based research outcomes. In this chapter, we summarize the ethical principles driving legislation and recommendations on animal care and use, as well as some of these laws and international recommendations. Examples of the impact of specific animal care and use aspects on research, as well as systems of internal and external oversight of animal care and use programs, are described.

\section{Keywords}

Animal care and use · Animal studies · Interplay · Preclinical data quality ·

Reliability

\section{$1 \quad$ Ethical and Legal Framework}

The use of animals for research purposes has been a subject of debate for a long time. The increase of societal concerns on this matter has been and is being reflected in the development and implementation of guidelines and strict legislation on the protection of animals used in research across the world (Guillen 2017). Although international legislation may differ between countries in some practical aspects, they are all based on the same ethical principles, mainly the Three Rs of Replacement, Reduction, and Refinement (Russell and Burch 1959) and, at a lesser extent, the Five Freedoms (Brambell 1965). The Three Rs are explicitly mentioned in most important international guidelines and regulations:

- Replacement refers to the avoidance or replacement of the use of animals in experiments where otherwise they would have been used. However, the concept of "relative replacement" based on strategies focused on reduction of animals and refinement of procedures performed on animals is also valid.

- Reduction refers to minimizing the number of animals needed to obtain the desired research objectives. Reduction strategies are normally based on improvements of the experimental design and/or the implementation of new techniques (e.g., imaging). An important concept is not only using less number of animals, but the right number, as using too low numbers could invalidate the research results.

- Refinement refers to the implementation of housing (e.g., micro- and macroenvironment), care (e.g., husbandry practices and veterinary care), and use (experimental techniques) procedures that minimize animal pain and distress.

The Five Freedoms (from hunger and thirst; from discomfort; from pain, injury, or disease; from fear and distress; and from the ability to express normal behavior), although initially proposed for the farm animal environment, are also referred to in some legislation on research animals, especially in Asia, but also have been recently considered for other related purposes, such as a recent proposal for the harm-benefit 
analysis to be performed as part of the ethical evaluation of research projects (Brønstad et al. 2016; Laber et al. 2016).

\subsection{Recommendations for the Care and Use of Laboratory Animals}

The common aspects of the ethical framework described above help with the harmonization of animal care and use in science, and of science itself through the implementation of international guidelines and recommendations, as well as compatible pieces of legislation (Guillen and Vergara 2017). Based on the current ethical concepts, the International Council for Laboratory Animal Science (ICLAS) collaborated with the Council for International Organizations for Medical Sciences (CIOMS) to update the International Guiding Principles for Biomedical Research Involving Animals, with the intention to guide emerging countries in developing a framework of responsibility and oversight on the use of animals in research and to serve as an international benchmark also in countries with well-developed animalbased research programs (Council for International Organizations for Medical Sciences and International Council for Laboratory Animal Science 2012). The Three Rs represent a significant aspect of this document. Also, the World Organization for Animal Health (OIE), with 180 member countries, recognizes the Five Freedoms as valuable guidance in animal welfare and more specifically describes the Three Rs and highlights their key role in the use of animals in science in Chap. 7.8 of the Terrestrial Animal Health Code (World Organization for Animal Health 2012).

One of the most widely followed sets of recommendations on animal care and use can be found in the Guide for the Care and Use of Laboratory Animals (Guide; National Research Council of the National Academy of Sciences 2011). The Guide, issued in the USA by the Institute for Laboratory Animal Research (ILAR) of the National Research Council (NRC), is the main nonregulatory reference in the USA, also serves as the basis for regulations and recommendations in other areas of the world (Guillen 2017), and is one of the primary standards for the accreditation of animal care and use programs across the world performed by the nonprofit organization AAALAC International (see below). The Guide refers to the Three Rs and to the US Government Principles for the Utilization and Care of Vertebrate Animals Used in Testing, Research, and Training (United States Government 1985), which already incorporate these same basic ethical principles. It offers recommendations on all areas of an animal care and use program, such as the institutional responsibilities (including the oversight process, the training of personnel, and the occupational health and safety program); the animal environment, housing, and management; the veterinary care; and the physical plant. The Guide states that it "is created by scientists and veterinarians for scientists and veterinarians to uphold the scientific rigor and integrity of biomedical research with laboratory animals as expected by their colleagues and society at large" and "establishes the minimum ethical, practice, and care standards for researchers and their institutions." 
Many professional organizations publish more specific recommendations on particular areas of an animal care and use program, such as ethical review, health monitoring, or education and training. Especially noteworthy are the Federation of European Laboratory Animal Science Associations (FELASA; www.felasa.eu), the American Association for Laboratory Animal Science (AALAS; www.aalas.org), and the Canadian Council on Animal Care (CCAC; www.ccac.ca). Other organizations focus exclusively on the development and dissemination of Three Rs initiatives, like the NC3Rs in the UK (www.nc3rs.org.uk), the North American 3Rs Collaborative in the USA (http://www.na3rsc.org/home.html), or Norecopa in Norway (https://norecopa.no/).

\subsection{Legislation in the USA}

In the USA, legislation on the care and use of animals comes from the US Department of Agriculture (USDA) and the Public Health Service (Bradfield et al. 2017). The Guide details the requirements of the Public Health Service Policy (PHS Policy) on Humane Care and Use of Laboratory Animals (Public Health Service 2002) and is used by institutions to comply with the PHS Policy. Therefore, although the Guide is not a piece of legislation, its standards are considered as minimum requirements by the PHS. On the USDA side, the Animal Welfare Act and related Animal Welfare Regulations represent the only federal law in the USA that regulates the treatment of animals in research, with the particularity that rats, mice, and birds are not considered regulated species (United States Government 1966). However, the Guide standards are applied to all vertebrate species. The US system gives a lot of autonomy to the institutions and is based on the Institutional Animal Care and Use Committees (IACUCs), where participation of researchers is mandatory. The IACUC is the body responsible for the evaluation and authorization of the research protocols, and the oversight of the entire institutional animal care and use program, including the appropriate training of personnel to perform the assigned tasks.

\subsection{Legislation in the European Union}

Legislation in the European Union is based on Directive 2010/63/EU (European Parliament and the Council of the European Union 2010), which has been transposed into the legislation of all European member states (Guillen et al. 2017). The Directive addresses explicitly the Three Rs and distributes the main responsibilities between the public competent authorities and the users. Authorized establishments must have an Animal Welfare Body (AWB) with an advisory function on ethical matters, while the (ethical) project evaluation is assigned to the public competent authorities. However, the Directive allows member states to designate other bodies than public competent authorities for the implementation of certain tasks, and at present the project evaluation is performed in a variety of manners across the European Union, either by institutional ethics committees, external bodies, public 
competent authorities at regional or national level, or a combination of them (Guillen et al. 2015). Annex III of the Directive dictates the requirements for care and accommodation of animals, based on the minimum cage sizes of the Appendix A of the European Convention ETS 123, which, although not a piece of legislation, was the first pan-European document addressing in detail the protection of animals in science and was signed and ratified by a majority of the members of the Council of Europe (Council of Europe 1986, 2006). These minimum cage sizes are generally bigger than the ones recommended in the Guide and represent one of the most visible differences between the US and European research programs. In terms of training of personnel, the Directive requires that staff shall be adequately educated and trained before they perform certain functions including carrying out procedures on animals and designing procedures and projects and that competence is demonstrated. However, it is a competence of the member states to establish the minimum training requirements.

The European Commission has published a number of consensus documents on the implementation of the Directive, which can be found at: http://ec.europa.eu/ environment/chemicals/lab_animals/interpretation_en.htm. Similar legal requirements are enforced in other European countries outside of the European Union, e.g., in Switzerland and Norway (Guillen et al. 2017; NORECOPA 2016).

\subsection{Legislation in Other Countries}

Other areas of the world have also developed legislation which have been extensively described elsewhere (Guillen 2017). In addition to countries such as Canada, Australia, New Zealand, or Israel having similar frameworks to those developed in the US or the European Union, many Asian countries have developed specific legislation, as well as several countries in Latin America (i.e., Brazil, Mexico, and Uruguay). Africa is the region where there is less legislation, although there are already professional associations or scientific events in some countries.

The most important aspect of the legislative initiatives is that all of them are based on the same ethical principles and try to achieve the same objectives (Guillen and Vergara 2017): Improving animal welfare standards in science is an objective per se, but this objective brings along another very important one which is the improvement of scientific quality.

Legislation that reflects international, common ethical questions is a key element in achieving these objectives. Also, legal documents normally address the same main topics: a process for the ethical evaluation (and authorization) of research protocols or projects; the need for appropriate training and competence of all personnel involved in the care and use of animals (caretakers, researchers, veterinarians, etc.); the animal environment and management (housing conditions, daily care, etc.); the need of effective veterinary care; and general requirements for facilities. Even in countries lacking specific legislation, researchers, veterinarians, and research institutions and associations work to follow these general instructions and the establishment of oversight systems, and the existence of active IACUCs or 
institutional ethics committees to review and improve the research protocols involving the use of animals is common also in these areas.

\section{Implications for Preclinical Data Quality}

\subsection{Oversight Bodies Impact on Preclinical Data Quality}

Thus, there is an extensive legal framework in many countries that regulates animal care and use, but how does animal care and use affect the quality of the preclinical data generated in animal experiments? First, the legal framework and its interpretation by oversight bodies (i.e., IACUCs/AWBs/ethics committees) can have a significant impact on data quality, both positive and negative.

As mentioned above, a reduction in the number of animals used according to the Three Rs is an important concept for the ethical evaluation of an animal study. But overemphasizing the need to minimize the number of animals in an experiment, without consideration of the appropriate number of animals needed to reliably answer the research question, can lead to underpowered studies with spurious results. Likewise, an uncritical refusal of study replications as unnecessary duplication of previous experiments by oversight bodies would violate the principles of good scientific methods required to gain confidence in an experimental finding (Pritt and Hammer 2017). A more balanced view by oversight bodies, on the other hand, helping with experimental design and statistical input to, e.g., determine the required sample size for the proposed studies at time of project application, can be an important step to ensure the appropriate number of animals is used and to facilitate the generation of reliable data. Clearly, this is what IACUCs/AWBs/ethical committees should strive for.

\subsection{Animal Care and Use Programs Affect Preclinical Data Quality}

Second, it is important to recognize that, despite the legal framework, animal facilities and their institutional animal care and use programs can differ in many aspects, even within the same country or the same organization (e.g., university). For example, there could be different barrier, hygiene, and sanitation levels to protect the health and well-being of the animals and the people working in the facility; animals could differ in microbiological status and receive different levels of veterinary care; there could be differences in the macroenvironmental (temperature, relative humidity, light intensity and duration, noise level, air circulation) and/or in the microenvironmental conditions (e.g., caging system, bedding, physical or social enrichment); there could be variations in food supplied and in water quality and also in a number of procedures, e.g., in animal acclimation, handling, transport, or surgery, to name a few, all of which could affect experimental outcome (Table 1). 
Table 1 Aspects of an animal care and use program that can affect the quality of preclinical data from animal studies

- Physical plant and environmental conditions (e.g., building material, control of environmental factors, such as temperature, relative humidity, air quality)

- Training (e.g., qualifications, experience, and competence of animal technicians, researchers, veterinarians)

- Oversight (internal, by IACUC/AWB/ethics committee; external, by competent authority; or third-party accreditation, AAALAC International, CCAC)

- Housing (e.g., caging system, space, enrichment, holding room)

- Husbandry (e.g., cleaning and sanitation, food, water, bedding)

- Animal procurement (e.g., source, transport)

- Quarantine and biosecurity practices

- Health monitoring program

- Veterinary interventions

- Surgical program (techniques, asepsis, anesthetic regimens, postsurgical care)

- Pain and distress (e.g., medication, recovery)

- Euthanasia method

\subsection{Health Status Influencing Preclinical Data}

Fox and colleagues, for example, reported on a study designed to determine whether long-term oral supplementation with creatine, used by athletes in training, would cause histologic organ lesions in mice. Animals treated with creatine developed hepatitis but so did the control mice. Notably, Helicobacter bilis ( $H$. bilis) was isolated from these mice and associated with hepatotoxicity seen in that study, thus confounding the experiment (Fox et al. 2004). A related Helicobacter species, $H$. hepaticus, has also been associated with hepatitis, inflammatory bowel disease, and cancer (Zenner 1999) and can promote drug-induced tumorigenesis in mice (Diwan et al. 1997; Nagamine et al. 2008; Stout et al. 2008). Of note, mouse Helicobacter infections often remain subclinical, and the animals appear healthy but can become symptomatic. The occurrence of clinical signs depends on various factors, such as strain, immunocompetency, and the gastrointestinal microbiome (Ihrig et al. 1999; Staley et al. 2009; Yang et al. 2013) and hence can lead to unexpected confounds of animal studies.

There are several other opportunistic and obligatory pathological microorganisms that can interfere with experimental outcome. It is therefore important that animals are regularly screened for the presence of these microorganisms (cf. FELASA recommendations for health monitoring in rodents, last revision: Mähler et al. 2014), either to exclude infected animals from the experiment, to initiate treatment if required (and possible), or at least to have clarity about the presence or absence of microorganisms.

However, the importance of microorganisms goes beyond agents causing clinical or subclinical disease. The gut microbiota also plays a critical role in animal and human health and disease, and its impact on animal physiology and, therefore, on how animals react in certain studies has been extensively studied in recent years 
(Franklin and Ericsson 2017; Hansen et al. 2015). Both the potential impact of infectious agents and natural microbiota can be modified by routine housing and husbandry conditions.

\subsection{The Impact of Housing and Husbandry}

Housing conditions and husbandry can also have substantial effects on experimental rodent data, yet often researchers are not fully aware of all the environmental factors in an animal facility that can influence data quality in their experiments (Jain and Baldwin 2003; Toth 2015). These factors include cage size, positioning of the holding cage in the rack, cage material, type of bedding, ambient cage temperature, humidity, noise levels, light intensity, duration of the light/dark cycle, number of animals per cage (individual vs. social housing), food access (continuous or restricted), type of food, physical enrichment provided, cage changing practices, transporting cages with animals within a room or between rooms, and sanitation cycle of the holding room (reviewed in Castelhano-Carlos and Baumans 2009; Everitt and Foster 2004; Nevalainen 2014; Toth 2015), and this list is far from complete.

Social housing, for example, increased dopamine D2 receptor expression in dominant monkeys, but not in subordinate monkeys, when compared to individual housing, and also affected the reinforcing properties of cocaine (Morgan et al. 2002). The stability of baseline cardiovascular parameters was affected by the arrangements of pens and the social setting in dogs implanted with telemetry devices (Klumpp et al. 2006). Similarly, social enrichment has been reported to affect cardiovascular function at resting state in monkeys (Xing et al. 2015). Housing temperature affects the growth rate of tumors in mice (Hylander and Repasky 2016; Kokolus et al. 2013). These examples highlight the importance of housing conditions on preclinical data across a variety of species.

Thus, the health status of the animal, environment factors in the animal facility, daily animal care routines and experimental manipulations (e.g., recovery surgery), as well as the experience, skills, and qualifications of the people performing these activities in the animal facility (animal care staff, veterinarians, researchers) contribute to the variability of preclinical data generated in animals (Howard 2002). A reduction in the variability of experimental data generated in laboratory animals has been coupled to refinements in microbial quality monitoring and husbandry, as well as higher professional expertise (Quimby 1993), and there is additional evidence suggesting that this trend continues with additional refinements, e.g., the introduction of environmental enrichment, even though this was initially much debated (Bayne and Würbel 2014). This is important, not only for the quality of the preclinical data generated but also from an ethical perspective as high data variability requires a higher number of test animals for a study to be conclusive. Also, the scientific utility of a highly variable and non-reproducible study can be questioned, with the associated risk that animals are wasted. As has been pointed out, "laboratory animal husbandry issues are an integral but [unfortunately still] 
underappreciated part of investigator's experimental design" (Nevalainen 2014, p. 392). Thus, there is a plea for even more reduction of variability in further refined animal care and use programs and for more detailed reporting of animal holding conditions in publications in order to enhance transparency and consequently reproducibility of preclinical data.

With this aim in mind, guidelines have been proposed for the items to be considered when planning and reporting animal experiments (see also chapter "Minimum Information in In Vivo Research"). The PREPARE Guidelines aim to help researchers to consider all relevant items when planning animal experiments "to reduce the risk of problems, artefacts or misunderstandings arising once studies have begun" (Smith et al. 2018). The ARRIVE Guidelines were developed "to maximize the output from research using animals by optimizing the information that is provided in publications on the design, conduct, and analysis of the experiments" (Kilkenny et al. 2010). Many scientific journals have already adhered to the ARRIVE Guidelines (although their impact is debatable, cf. Hair et al. 2019) and more recently an update of the guidelines has been published (Percie du Sert et al. 2019a, b), while the impact of the PREPARE Guidelines is still to be evaluated.

\section{Assessment of Animal Care and Use Programs}

\subsection{Internal Oversight}

Internal oversight bodies, i.e., IACUCs/AWBs/ethics committees, can have a significant impact on data quality. First, they are tasked with the review of the ethical protocols, in accordance with national and international legislation and institutional policies. As part of this review process, the internal oversight body also plays an important role in advising on the experimental design of the proposed studies, including statistical considerations, and assures pain, discomfort, and distress are reduced to a minimum (Everitt and Berridge 2017; Silverman et al. 2017). As mentioned above, those factors can significantly impact on preclinical data quality. Second, the internal oversight body should conduct inspections of its own animal program and facility, at least annually or preferentially more often, also depending on legislation and policies. Besides assurance of the ethical and humane use of animals in research, this will also ensure that all aspects of an animal care and use program that can affect the quality of preclinical data from animal studies are well controlled, and it will create opportunities for further improvements of the quality of research, e.g., by assuring that surgical facilities are state of the art and that investigators conducting surgical procedures are properly trained. Third, postapproval monitoring conducted by the oversight body, primarily serving to ensure that animal use is occurring as described in the approved protocol, may also contribute to data quality. There are interdependencies between compliance, consistency, and reproducibility, and failure to reproduce an experiment has been considered as an unintended consequence of noncompliance with approved procedures (Silverman et al. 2017). Thus, the internal oversight body plays a pivotal role in the assurance of data quality in animal studies. 


\subsection{External Oversight}

Depending on national legislation, ethical evaluation and/or authorization for animal studies may also be provided by external ethics committees, e.g. by bodies at the regional or national competent authority level, or other bodies authorized by the competent authorities to perform the ethical evaluation on their behalf. Their role in the assurance of data quality during project review is comparable to the role of an internal oversight body. In addition, many countries have a mandatory requirement for regular, announced or unannounced, inspections of animal facilities by a competent authority to monitor compliance with legal obligations. Naturally, experienced inspectors will also have an impact on preclinical data quality through assurance of a compliant animal care and use program. However, often these inspections are riskbased and may not cover all aspects of an animal care and use program that could affect preclinical data quality, and whether advice relevant to data quality is given may also depend on the profile, skills, and experience of the individual inspector. The internal oversight body is much better positioned to ensure full coverage of the aspects relevant to the quality of data from studies involving animals, to promote consistency and timely action, if required, and should take primary responsibility.

\subsection{The AAALAC International Accreditation Process}

AAALAC International (AAALAC) (www.aaalac.org) is a voluntary accrediting organization that enhances the quality of research, testing, and education by promoting humane and responsible research animal care and use through provision of advice and independent assessments to participating institutions and accreditation of those that meet or exceed applicable standards. More than 1,000 institutions including companies, universities, hospitals, government agencies, and other research institutions in 47 countries have earned AAALAC accreditation, demonstrating their commitment to responsible animal care and use. These institutions volunteer to participate in AAALAC's program, in addition to complying with the implementing laws that regulate animal research.

AAALAC was established in 1965 in the USA and is governed by approximately 70 scientific organizations from all around the world. The assessment and accreditation activities are performed by independent professionals with expertise in the field, who form the Council on Accreditation. The Council has three North American sections, two in the Pacific Rim, and one in Europe, each taking care of the activities in their respective geographical areas. The primary standards used by the Council are the Guide, the ETS 123, and the Guide for the Care and Use of Agricultural Animals in Research and Teaching (Federation of Animal Science Societies 2010). The Council may also use other scientific publications on different topics called Reference Resources (https://www.aaalac.org/accreditation/resources.cfm) and has to ensure that accredited programs comply with the implementing legislation in the specific location of the evaluated program. Council members are helped by ad hoc consultants/specialists, who are the same type of professionals, normally selected based on the particular expertise needed for each evaluation process. 
When one institution voluntarily applies for the accreditation, it has to complete and submit the Program Description (https://www.aaalac.org/accreditation/apply. $\mathrm{cfm})$, a document where all areas of the animal care and use program have to be thoroughly described. This includes the institutional responsibilities (key responsible personnel, oversight and ethical review process, competence of personnel, and occupational health and safety program), the animal environment, housing and management, the veterinary care program, and the physical plant. The Program Description is then reviewed by a Council member and the collaborating ad hoc(s), and a site visit to the institution is scheduled to evaluate the quality of the program on site. The report coming from this site visit is reviewed by and discussed with the other Council members of the same section and a decision on the accreditation status taken. Depending on the severity of the issues (if any) identified during the process, there may be mandatory issues that the institution must correct before obtaining full accreditation, and/or suggestions for improvement, which are strong recommendations for the improvement of the program that the institution can voluntarily address.

The evaluation process is based on performance standards rather than on engineering standards, which is particularly important when considering the global scope of AAALAC (Guillen 2012). While engineering standards are rigidly defined, easily measurable (e.g., minimum cage sizes), performance standards are outcome oriented, focused on goals or expected results rather than the process used to achieve the results, and have the flexibility needed in the diverse research environment. AAALAC has to make sure that institutions comply with the engineering standards which are normally part of legislation, but on top of that also apply the performance standards as described in the AAALAC Primary Standards. For example, AAALAC may accept different ethical review processes if they, in addition to be legally compliant, are effective and there is evidence of a good outcome.

The AAALAC accreditation process is compatible with quality systems like GLP or ISO. In fact, many institutions who implement GLP or ISO because they perform regulated research or have general quality systems in place (e.g., contract research organizations, pharmaceutical companies) also implement the AAALAC accreditation as this is the only global system specifically focused on animal care and use programs and carried out by independent professionals in the field. This peer-review process has been extremely successful and continues to expand in institutions around the world.

\subsection{Assessments by Industry}

Animal studies form an integral part of the drug development process. Those studies are either conducted within the research facilities of a company or are outsourced and performed by external service providers. To ensure external partners comply with technical requirements and ethical standards, more and more pharmaceutical companies started to formally assess the animal care and use programs of their collaborators on a regular basis, including contract research organizations (CROs), 
academic groups, and breeders (Mikkelsen et al. 2007; Underwood 2007). A more recent development is the joint assessment of breeders and CROs by consortia of pharmaceutical companies, which facilitates harmonization of processes across companies and enhances capacity and expertise (Interpharma 2018). In general, these animal care and use program assessments cover the aspects highlighted in Table 1, plus additional topics, such as documentation, occupational health, and safety, and often are closely oriented on the AAALAC process.

\section{Conclusion}

There are multiple evidences of the influence of animal care and use conditions and practices on animal-based research outcomes. The existing legislation on the use of animals in research, established upon internationally accepted ethical principles, helps creating a more common research environment that facilitates extrapolation of research results obtained in particular institutions. However, animal care and use practices may differ significantly across institutions, with potentially significant and often unknown effects on research results. Professional science-based recommendations try to complement legislation by creating standards on a number of areas, including ethical review, health monitoring, animal environment, husbandry practices, training of personnel, and others. But the implementation of the standards by research institutions still varies significantly and is very often depending on institutional or even individual commitment.

What can we learn for Good Research Practice? First and foremost, it should be clear now that the quality of animal care and use directly impacts on the quality of preclinical data. In addition, the field of animal care and use has established a framework that could be seen as a role model for Good Research Practice: Minimum requirements as defined by guidelines and legislation such as the Guide or the Directive 2010/63/EU set the standards for animal care and use programs in the USA and in EU member states, and both internal and external oversight bodies have been created to ensure proper implementation and adherence to these standards. The review and oversight of animal care and use programs is a key tool to not only ensure compliance with legal requirements but also to establish a well-defined research environment that considers all aspects of animal care and use that can impact research outcomes. This oversight may be internal, already mandated by legislation in many countries, and external by peers. A combination of the day-to-day internal oversight with a periodic independent external review seems to be the most efficient way to ensure the implementation of a high-quality animal care and use program where, in addition to addressing animal ethical and welfare issues, researchers can produce better quality science. 
Neither the Guide nor the Directive 2010/63/EU is overtly prescriptive (except for a few clearly defined and nonnegotiable engineering standards), and also accrediting organizations such as AAALAC International strongly adhere to the principle of performance standards, which allows flexibility in the implementation of these standards.

These principles of minimum requirements, performance standards, and internal and external oversight could be implemented in other areas of research in a manner that is fit for the intended purpose. One may envision Good Research Practice that is guided by lean, easy to use minimal requirements defined by a quality system, based on the specific needs of the research group and working on performance standards, with day-to-day internal oversight and periodic external assessments, not to police but to improve daily research practice, possibly in combination with an accreditation process. The European Quality in Preclinical Data (EQIPD) IMI consortium (https:// quality-preclinical-data.eu/) is in fact following these same principles, with the added advantage to look at animal care and use and Good Research Practice holistically, as not only does animal care and use affect the quality of preclinical data, but the need for preclinical data quality will impact on animal care and use.

\section{References}

Bayne K, Würbel H (2014) The impact of environmental enrichment on the outcome variability and scientific variability of laboratory animal studies. Rev Sci Tech 33:273-280

Bradfield JF, Bennet BT, Gillett CS (2017) Oversight of research animal welfare in the United States. In: Guillen J (ed) Laboratory animals: regulations and recommendations for the care and use of animals in research. Academic Press, Cambridge, pp 15-68

Brambell R (1965) Report of the technical committee to enquire into the welfare of animals kept underintensive livestock husbandry systems, cmd. HM Stationery Office, London, pp 1-84

Brønstad A, Newcomer CE, Decelle T, Everitt JI, Guillen J, Laber K (2016) Current concepts of harm-benefit analysis of animal experiments - report from the AALAS-FELASA working group on harm-benefit analysis - part 1. Lab Anim 50(1S):1-20

Castelhano-Carlos MJ, Baumans V (2009) The impact of light, noise, cage cleaning and in-house transport on welfare and stress of laboratory rats. Lab Anim Sci 43:311-327

Council for International Organizations for Medical Sciences and International Council for Laboratory Animal Science (2012) International guiding principles for biomedical research involving animals. www.iclas.org

Council of Europe (1986) European convention for the protection of vertebrate animals used for experimental and other scientific purposes. Eur Treat Ser 1986:123

Council of Europe (2006) Appendix A of the European convention for the protection of vertebrate animals used for experimental and other scientific purposes (ETS No. 123). Guidelines for accommodation and care of animals (Article 5 of the convention). Approved by the multilateral consultation. Cons 123:3

Diwan BA, Ward JM, Ramliak D, Anderson LM (1997) Promotion by helicobacter hepaticusinduced hepatitis of hepatic tumors initiated by n-nitrosodimethylamine in male $\mathrm{A} / \mathrm{JCr}$ mice. Toxicol Pathol 25:597-605 
European Parliament and the Council of the European Union (2010) Directive 2010/63/EU of the European Parliament and of the Council of 22 September 2010 on the protection of animals used for scientific purposes. Off J Eur Union 2010, L 276:33-79

Everitt JI, Berridge BR (2017) The role of the IACUC in the design and conduct of animal experiments that contribute to translational success. ILAR J 58:129-134

Everitt JI, Foster PMD (2004) Laboratory animal science issues in the design and conduct of studies with endocrine-active compounds. ILAR J 45:417-424

Federation of Animal Science Societies (2010) Guide for the care and use of agricultural animals in research and teaching, 3rd edn. Federation of Animal Science Societies, Champaign

Fox JG, Rogers AB, Whary MT, Taylor NS, Xu S, Feng Y, Keys S (2004) Helicobacter bilisassociated hepatitis in outbred mice. Comp Med 54:571-577

Franklin CL, Ericsson AC (2017) Microbiota and reproducibility of rodent models. Lab Anim 46 (4):114-122

Guillen J (2012) Accreditation of animal care and use programmes: the use of performance standards in a global environment. Anim Technol Welfare 11(2):89-94

Guillen J (2017) Laboratory animals: regulations and recommendations for the care and use of animals in research. Academic Press, Cambridge

Guillen J, Vergara P (2017) Global guiding principles: a tool for harmonization. In: Guillen J (ed) Laboratory animals: regulations and recommendations for the care and use of animals in research. Academic Press, Cambridge, pp 1-13

Guillen J, Robinson S, Decelle T, Exner C, Fentener van Vlissingen M (2015) Approaches to animal research project evaluation in Europe after implementation of directive 2010/63/EU. Lab Anim 44(1):23-31

Guillen J, Prins JB, Howard B, Degryse AD, Gyger M (2017) The European framework on research animal welfare regulations and guidelines. In: Guillen $\mathrm{J}(\mathrm{ed})$ Laboratory animals: regulations and recommendations for the care and use of animals in research. Academic Press, Cambridge, pp 117-202

Hair K, Macleod MR, Sena ES, on behalf of the IICARus Collaboration (2019) A randomised controlled trial of an intervention to improve compliance with the ARRIVE guidelines (IICARus). Res Int Peer Rev 4:1-17

Hansen AK, Krych L, Nielsen DS, Hansen CHF (2015) A review of applied aspects of dealing with gut microbiota impact on rodent models. ILAR J 56(2):250-264

Howard BR (2002) Control of variability. ILAR J 42:194-201

Hylander BL, Repasky EA (2016) Thermoneutrality, mice, and cancer: a heated opinion. Trends Cancer 2:166-175

Ihrig M, Schrenzel MD, Fox JG (1999) Differential susceptibility to hepatic inflammation and proliferation in AXB recombinant inbred mice. Am J Pathol 155:571-582

Interpharma (2018) Animal welfare report 2018. https://www.interpharma.ch/1553-animal-wel fare-report-2018

Jain M, Baldwin AL (2003) Are laboratory animals stressed by their housing environment and are investigators aware that this stress can affect physiological data? Med Hypoth 60:284-289

Kilkenny C, Browne WJ, Cuthill IC, Emerson M, Altman DG (2010) Improving bioscience research reporting: the ARRIVE guidelines for reporting animal research. PLoS Biol 8(6):e1000412

Klumpp A, Trautmann T, Markert M, Guth B (2006) Optimizing the experimental environment for dog telemetry studies. J Pharmacol Toxicol Methods 54:141-149

Kokolus KM, Capitano ML, Lee CT, Eng JWL, Waight JD, Hylander BL, Sexton S, Hong CC, Gordon CJ, Abrams SI, Repasky EA (2013) Baseline tumor growth and immune control in laboratory mice are significantly influenced by subthermoneutral housing temperatura. Proc Natl Acad Sci U S A 110:20176-20181 
Laber K, Newcomer CE, Decelle T, Everitt JI, Guillen J, Brønstad A (2016) Recommendations for addressing harm-benefit analysis and implementation in ethical evaluation - report from the AALAS-FELASA working group on harm-benefit analysis - part 2. Lab Anim 50(1S):21-42

Mähler M, Berard M, Feinstein R, Gallagher A, Illgen-Wilcke B, Pritchett-Corning K, Raspa M (2014) FELASA recommendations for the health monitoring of mouse, rat, hamster, Guinea pig and rabbit colonies in breeding and experimental units. Lab Anim 48:178-192

Mikkelsen LF, Hansen HN, Holst L, Ottesen JL (2007) Setting global standards for animal welfare monitoring of external contractors. AATEXX 14:731-733

Morgan D, Grant KA, Gage HD, Mach RH, Kaplan JR, Prioleau O, Nader SH, Buchheimer N, Ehrenkaufer RL, Nader MA (2002) Social dominance in monkeys: dopamine D2 receptors and cocaine self-administration. Nat Neurosci 5:169-174

Nagamine CM, Rogers AB, Fox JG, Schauer DB (2008) Helicobacter hepaticus promotes azoxymethane initiated colon tumorigenesis in BALB/c-IL10-deficient mice. Int $\mathrm{J}$ Cancer 122:832-838

National Research Council of the National Academy of Sciences (2011) Guide for the care and use of laboratory animals. National Academy Press, Washington

Nevalainen T (2014) Animal husbandry and experimental design. ILAR J 55:392-398

NORECOPA (2016) EUs Directive 2010/63/EU on the protection of animals used for scientific purposes. https://norecopa.no/legislation/eu-directive-201063

Percie du Sert N, Hurst V, Ahluwalia A, Alam S, Avey MT, Baker M, Browne WJ, Clark A, Cuthill IC, Dirnagl U, Emerson M, Garner P, Holgate ST, Howells DW, Karp NA, Lidster K, MacCallum CJ, Macleod M, Petersen O, Rawle F, Reynolds P, Rooney K, Sena ES, Silberberg SD, Steckler T, Würbel H (2019a) The ARRIVE guidelines 2019: updated guidelines for reporting animal research. bioRxv. https://www.biorxiv.org/content/10.1101/703181v1

Percie du Sert N, Hurst V, Ahluwalia A, Alam S, Avey MT, Baker M, Browne WJ, Clark A, Cuthill IC, Dirnagl U, Emerson M, Garner P, Holgate ST, Howells DW, Karp NA, Lidster K, MacCallum CJ, Macleod M, Petersen O, Rawle F, Reynolds P, Rooney K, Sena ES, Silberberg SD, Steckler T, Würbel H (2019b) Reporting animal research: explanation and elaboration for the ARRIVE guidelines 2019. bioRxv. https://www.biorxiv.org/content/10.1101/703355v1

Pritt SL, Hammer RE (2017) The interplay of ethics, animal welfare, and IACUC oversight on the reproducibility of animal studies. Comp Med 67:101-105

Public Health Service (2002) Public health service policy on humane care and use of laboratory animals. Office of Laboratory Animal Welfare, National Institutes of Health, Public Health Service, Bethesda

Quimby FW (1993) Twenty-five years of progress in laboratory animal science. Lab Anim 28:158-171

Russell WMS, Burch RL (1959) The principles of humane experimental technique. Universities Federation for Animal Welfare, Potters Bar

Silverman J, Macy J, Preisig PA (2017) The role of the IACUC in ensuring research reproducibility. Lab Anim 46:129-135

Smith J, Clutton RE, Lilley E, Hansen KEA, Brattelid T (2018) PREPARE: guidelines for planning animal research and testing. Lab Anim 52(2):135-141

Staley EM, Schoeb TR, Lorenz RG (2009) Differential susceptibility of P-glycoprotein deficient mice to colitis induction by environmental insults. Inflamm Bowel Dis 15:684-696

Stout MD, Kissling GE, Suárez FA, Malarkey DE, Herbert RA, Bucher JR (2008) Influence of helicobacter hepaticus infection on the chronic toxicity and carcinogenicity of triethanolamine in B6C3F1 mice. Toxicol Pathol 36:783-794

Toth LA (2015) The influence of cage environment on rodent physiology and behavior: implications for reproducibility of pre-clinical rodent research. Exp Neurol 270:72-77 
Underwood W (2007) Contracting in vivo research: what are the issues? J Am Assoc Lab Anim Sci 46:16-19

United States Government (1966) Animal welfare act of 1966 (pub L. 89-544) and subsequent amendments. U.S.Code. Vol. 7, secs. 2131-2157 et seq

United States Government (1985) Principles for the utilization and care of vertebrate animals used in testing, research and training. OER Home Page - Grants Web, Office of Laboratory Animal Welfare: PHS policy on humane care and use of laboratory animals. http://grants.nih.gov/grants/ olaw/references/phspol.htm\#USGovPrinciples

World Organization for Animal Health (2012) Use of animals in research and education. Terrestial animal health code. https://www.oie.int/fileadmin/Home/eng/Health_standards/tahc/current/ chapitre_aw_research_education.pdf

Xing G, Lu J, Hu M, Wang S, Zhai L, Schofield J, Oldman K, Adkins D, Yu H, Ren J, Skinner M (2015) Effects of group housing on ECG assessment in conscious cynomolgus monkeys. J Pharmacol Toxicol Methods 75:44-51

Yang I, Eibach D, Kops F, Brenneke B, Woltemate S, Schulze J, Bleich A, Gruber AD, Muthupalani S, Fox JG, Josenhans C, Suerbaum S (2013) Intestinal microbiota composition of interleukin-10 deficient C57BL/6J mice and susceptibility to helicobacter hepaticus-induced colitis. PLoS One 9:e70783. https://doi.org/10.1371/journal.pone.0070783

Zenner L (1999) Pathology, diagnosis and epidemiology of the rodent helicobacter infection. Comp Immunol Microbiol Infect Dis 22:41-61

Open Access This chapter is licensed under the terms of the Creative Commons Attribution 4.0 International License (http://creativecommons.org/licenses/by/4.0/), which permits use, sharing, adaptation, distribution and reproduction in any medium or format, as long as you give appropriate credit to the original author(s) and the source, provide a link to the Creative Commons licence and indicate if changes were made.

The images or other third party material in this chapter are included in the chapter's Creative Commons licence, unless indicated otherwise in a credit line to the material. If material is not included in the chapter's Creative Commons licence and your intended use is not permitted by statutory regulation or exceeds the permitted use, you will need to obtain permission directly from the copyright holder. 